\title{
Construção e evidências de validade da Escala de Satisfação Corporal e Satisfação Sexual para Lésbicas (ESCSS-Lésbicas)
}

\author{
Juliana Fernandes-Eloi ${ }^{1}$ \\ Luciana Maria Maia ${ }^{2}$ \\ Elder Cerqueira-Santos ${ }^{3}$ \\ ${ }^{1}$ Universidade de Fortaleza, CE, Brasil \\ https://orcid.org/0000-0001-9036-8850 \\ ${ }^{2}$ Universidade de Fortaleza, CE, Brasil \\ https://orcid.org/0000-0003-1491-5685 \\ ${ }^{3}$ Universidade Federal de Sergipe, SE, Brasil \\ https://orcid.org/0000-0003-1116-6391
}

\begin{abstract}
Resumo
Este estudo apresenta as etapas de construção de um instrumento psicométrico específico para a população lésbica e a descrição de suas evidências de validação. Os dados quantitativos foram coletados no aplicativo online Google Forms, do Google Drive, por meio da amostra de 1.231 mulheres lésbicas brasileiras $(M=27,87 ; D P=9,10)$. Para a construção e validação do instrumento foram realizadas cinco etapas: 1) Avaliação qualitativa dos instrumentos pelo grupo focal com experts; 2) Avaliação dos instrumentos pelas informantes-chave; 3) Análise por juízes-pares; 4) Análise Fatorial Exploratória (AFE); e 5) Análise Fatorial Confirmatória (AFC). Os resultados da AFE e da AFC foram conduzidos no software Mplus versão 7.11. Os resultados descrevem a criação e as evidências de validade da ESCSSLésbicas, escala tipo Likert de 0 a 6, composta por 30 itens, e confiabilidade interna de 0,907. O instrumento mostrou-se adequado para a mensuração dos aspectos relativos à satisfação corporal e sexual de mulheres lésbicas.
\end{abstract}

Palavras-chave: lésbicas, satisfação corporal, satisfação sexual, evidências de validade, propriedades psicométricas.

\section{Construction and Validity Evidence of The Bodily Satisfaction and Sexual Satisfaction for Lesbians (LESBIAN-ESCSS)}

\begin{abstract}
The study presents the stages of a psychometric instrument construction specific to lesbian population and the description of their validation evidences. The quantitative data were collected the Google Forms app on google drive through the sample 1231 Brazilian lesbian women $(M=27,87 ; S D=9,10) .5$ steps were taken to the construction and validation of the psychometric instrument: 1) Qualitative evaluation of the instruments by focus group of experts; 2) Evaluation of instruments by key informants; 3) Analysis by peer judges; 4) Exploratory Factorial Analysis (EFA); 5) Confirmatory Factorial Analysis (CFA). The results of the EFA and the CFA were conducted in Mplus software version 7.11. The results describe the creation and validation evidences of the BSSSS-Lesbian, scale Likert type from 0 to 6 , composed by 30 items, and the internal reliability of the whole scale is 0.907 . The instrument was specific and adequate to the measurement of aspects related to the body satisfaction and sexual in lesbian women.
\end{abstract}

Keywords: lesbian, body satisfaction, sexual satisfaction, evidence of validity, psychometric properties. 


\title{
Construcción y Evidencias de Validez de la Escala de Satisfacción Corporal Y Satisfacción Sexual Para Lésbicas (ESCSS-Lésbicas)
}

\begin{abstract}
Resumen
Este estudio presenta las etapas de construcción de un instrumento psicométrico específico para la población lésbica y la descripción de sus evidencias de validación. Los datos cuantitativos fueron recolectados en la aplicación en línea Google Forms de Google Drive utilizando una muestra de 1.231 mujeres lesbianas brasileñas $(M=27,87 ; D E=9,10)$. Para la construcción y validación del instrumento psicométrico específico para la población lésbica se realizaron 5 etapas: 1) Evaluación cualitativa de los instrumentos por el grupo focal expertos; 2) evaluación de los instrumentos por las informantes clave; 3) Análisis por jueces pares; 4) Análisis Factorial Exploratorio (AFE) y 5) Análisis Factorial Confirmatorio (AFC). Los resultados del análisis factorial exploratorio y del análisis factorial confirmatorio fueron realizados en el software Mplus versión 7.11. Los resultados describen la creación y las evidencias de validez de la ESCSS-Lesbianas, escala compuesta por 30 ítems tipo Likert de 0 a 6, la confiabilidad interna (alfa de Cronbach) de la escala total es de 0,907. El instrumento se mostró específico y adecuado para la medición de los aspectos relativos a la satisfacción corporal y sexual de mujeres lesbianas.
\end{abstract}

Palabras clave: lesbianas, satisfacción corporal, satisfacción sexual, evidencias de validez, propiedades psicométricas.

Discutir propriedades psicométricas de ferramentas que avaliem a sexualidade se apresenta como fenômeno complexo, e problematizações que abarcam posicionamentos muitas vezes ideológicos, políticos e até fundamentalistas, dificultam ainda mais um criterioso posicionamento em relação aos estudos da sexualidade. Nesse sentido, analisar e mensurar aspectos pertinentes à sexualidade exige uma maior articulação entre processos culturais, psicológicos e sociais que perpassam a sexualidade e, neste trabalho em especifico, a sexualidade de mulheres lésbicas (Gato, Fontaine, \& Leme, 2014; Lingiardi, Baiocco \& Nardelli, 2012; Ross \& Rosser, 1996; Shidlo, 1994).

Para Gato, Fontaine e Leme (2014), embora a negação de diferentes expressões da sexualidade ainda seja fenômeno presente, se reconhece nos dias atuais e em sociedades mais desenvolvidas uma maior expansão dos direitos cíveis de pessoas lésbicas, gays, bissexuais, transexuais, travestis, transgêneros, queer e intersexuais (LGBTTTQI's). Contudo, a ausência de informações ainda sustenta estigmas sociais que condenam e discriminam diferentes orientações sexuais (Cerqueira-Santos, Winter, Salles, Longo, \& Teodoro, 2007; Nogueira \& Oliveira, 2010).

Até a primeira metade do século XX, se articulava a orientação sexual ou até mesmo a própria sexualidade sob a fundamentação de uma perspectiva binária, descrita, sobretudo, por comportamentos e afetos sexuais entre homens e mulheres na sociedade, nas escolas e igrejas (Haraway, 2004). Muitos movimentos sociais após a elaboração da perspectiva binária se articularam socialmente, como por exemplo, a organização dos movimentos de mulheres e feministas, priorizando a equidade de gênero e o direito as práticas políticas, sexuais e econômicas.

Na verdade, os movimentos de mulheres e os movimentos feministas surgiram como reação coletiva, sendo um produto de resistência contra a cultura patriarcal potencializando discussões acerca da orientação sexual em todo o mundo (Duby \& Perrot, 1998; Fernandes, 2012; Nogueira \& Oliveira, 2010). A orientação sexual passa a ser um fenômeno mais discutido, mas mesmo assim, ainda permanece como conceito considerado tabu.

Todavia, pode-se pensar a orientação sexual como um elemento da identidade sexual, a qual é composta por uma série de fatores pertencentes às questões biológicas, psíquicas, sociais e sexuais. Parte-se ainda do pressuposto que, para além de uma opção ou alternativa de escolha, os indivíduos estão inseridos em contextos objetivos, subjetivos e fisiológicos que irão, a partir de um sistema identitário, possibilitar a estruturação de atrações, fantasias, desejos eróticos e sexuais.

Orientação sexual, portanto, envolve relações, emoções, que podem ser vividas em direção ao sexo oposto, ao mesmo sexo e/ou por ambos os sexos (APA, 2008). Por exemplo, é preciso reconhecer as variabilidades 
das identidades sexuais lésbicas, em que a experiência de mulheres lésbicas é qualitativamente diferente das experiências vividas por mulheres héteros ou bissexuais (Fernandes, Barroso, Assis, \& Pocahy, 2015). Configurase um fenômeno de maior e mais intensa discriminação, implicando o desafio de viver como lésbica em contexto opressor, que pode favorecer aspectos de resiliência na geração de estratégias frente a situações de risco e vulnerabilidades sociais e jurídicas. Nesse sentido, compreende-se que a satisfação sexual dessa população passa por esses aspectos e sofre variações dada a condição de invisibilidade vivida na sexualidade lésbica.

\section{Sexualidade e satisfação sexual}

A sexualidade humana possui uma variabilidade dinâmica, subjetiva e polimorfológica em que a orientação sexual, por sua vez, se apresenta como constitucional e não uma questão de escolha ou modismo. Ao se estabelecer em relações possivelmente vinculares, com atrações físicas, e sentimentais, a orientação sexual se apresenta de modo complexo e distinto dos comportamentos sexuais (APA, 2008).

O comportamento sexual, por sua vez, se apresenta como gesto ou atitude frente à realização de práticas e tarefas sexuais, sem necessariamente possuírem caráter identitário (O’Murray, 2002). Os comportamentos homossexuais ou heterossexuais apresentam diferentes possibilidades, não fixadas, que se referem às representações comportamentais, podendo ou não, serem vividos no âmbito dos desejos sexuais. Ou seja, parecer ou possuir estereótipos de uma mulher lésbica não significa possuir desejos homossexuais, assim como demonstrar traços heterossexuais, em nada podem confirmar uma perspectiva heterossexual ou em relação a suas satisfações corporais e sexuais.

Até na existência do desejo e da atração sexual, não se pode confirmar a prática comportamental, pois "o corpo físico, no entanto, não é a totalidade do corpo, nem mesmo o corpo, a totalidade da pessoa" (Cardoso, 1994, p. 174). Estamos diante de uma rede integrada a diversos sistemas em que a sexualidade é o fio que perpassa todas as particularidades da composição da existência humana que incluem corpos, comportamentos, pensamentos, sentimentos e crenças, pois a construção da percepção e satisfação corporal ocorre por influência de contextos culturais, sociais e psicológicos (Lee, Damhorst, \& Ogle, 2009).

A sexualidade e a satisfação sexual que giram em torno da saúde sexual do indivíduo, ainda são ofuscados pela escassez de produção científica no Brasil. Contudo, cada vez mais, a sexualidade se apresenta como um dos parâmetros mais diretamente relacionados à qualidade de vida (Carvalheira \& Leal, 2008; Sprecher, Christopher, Cate, Vangelisti, \& Perlman, 2006; Wainberg, Hutz, \& Stenert, 2009). Ou seja, "since sexual satisfaction is an important health marker, to affect a woman's sexuality means to indirectly influence her quality of life"/ "uma vez que a satisfação sexual é um importante marcador de saúde, afetar a sexualidade de uma mulher significa influenciar indiretamente sua qualidade de vida" (Monteiro et al., 2016, p. 562).

Neste sentido, a satisfação sexual representa um importante fenômeno a ser estudado. Entretanto, há uma intensa dificuldade em adotar um único significado (Pechorro, Diniz, \& Vieira, 2009). A literatura tem mostrado diferentes abordagens de estudo em relação à temática. Ainda podem-se encontrar estudos que limitam a sexualidade a simples funcionalidade da vida sexual, não contemplando aspectos subjetivos, afetivos e emocionais atrelados à composição de toda trajetória sexual do indivíduo (Monteiro et al., 2016). Com menor frequência, encontram-se modelos teóricos que contemplam os aspectos psicológicos, orgânicos e físicos envolvidos na satisfação sexual (Wainberg, Hutz, \& Stenert, 2009). A satisfação sexual, por sua vez, não é somente função sexual, é uma dimensão fundamental para os estudos acerca da saúde sexual, psicológica e social do indivíduo (Catão, Rodrigues, Viviani, Finotelli Jr., \& Silva, 2010; Henderson, Lehavot, \& Simoni, 2009). 
Em outros países, escalas têm sido desenvolvidas para medir a satisfação sexual, como por exemplo, a Sexual Interaction Inventory (LoPiccolo \& Steger, 1974); Derogatis Sexual Functioning Inventory (Derogatis \& Melisaratos, 1979); Pinney Sexual Satisfaction Inventory (Pinney, Gerrard, \& Denney, 1987). Mais recentemente, outros instrumentos que medem a satisfação sexual são: Hudson Index of Sexual Satisfaction que contém 25 itens (Hudson, 1998) e outra escala é a Whitley Sexual Satisfaction Inventory que inclui itens para avaliação do grau de satisfação sexual em diferentes tipos de atividade sexual (Whitley, 1998).

Já no Brasil, na década de 1990, estudo realizado por Furlanetto e Rodrigues (1996) pesquisou acerca da satisfação sexual em 110 mulheres, com idades entre 25 e 40 anos e que estivessem em relacionamentos fixos. Para a coleta de dados, utilizaram um questionário e concluíram que $80 \%$ das mulheres pesquisadas associam satisfação sexual ao orgasmo; $74 \%$ das mulheres precisam sentir atração sexual pelo parceiro para ter satisfação sexual; $66 \%$ relatam que para sentirem satisfação sexual é fundamental a vivência de uma relação com parceiro carinhoso; e $85 \%$ das mulheres que responderam à pesquisa se sentem satisfeitas sexualmente.

Outro estudo, realizado por Meston e Trapnell (2005), objetivou desenvolver uma medida que avaliasse a satisfação e sofrimento sexual em mulheres. Para tanto, os autores aplicaram a Escala de Satisfação Sexual para Mulheres (SSS-W) em cerca de 800 participantes. A pesquisa se deu em três fases: 1) triagem de itens de acordo com a literatura, realização da análise fatorial exploratória e entrevista com 539 mulheres estudantes de psicologia; 2) a alteração do questionário, aperfeiçoamento dos itens e análises fatorial, aplicado em 119 mulheres; e 3) alteração final do questionário aplicado a uma amostra de 102 mulheres com disfunção sexual clinicamente diagnosticadas, e em 79 mulheres com nenhuma disfunção sexual. O SSS-W é um instrumento que possui 30 itens de satisfação sexual e sofrimento sexual, composta por cinco domínios suportados por análises fatoriais: contentamento, comunicação, compatibilidade, preocupação relacional e preocupação pessoal. A aplicação da SSS-W confirmou evidência psicométrica de confiabilidade e se mostrou instrumento adequado na avaliação de mulheres com disfunção sexual.

A pesquisa realizada por Cardoso et al., (2009) buscou identificar e comparar aspectos da satisfação sexual, corporeidade e sexualidade entre homens e mulheres com diferentes orientações sexuais e de gênero. Para tanto, aplicou o Questionário de Identidade Corporal (QIC) em 108 participantes, que estavam no I Floripa Diversity Games, realizado na Universidade do Estado de Santa Catarina. O QIC possui 10 escalas: intimidade corporal, percepção corporal, satisfação corporal, pré-disposição sexual, comportamento sexual, orientação sexual, satisfação sexual, experiências motoras, orientação motora e identidade infantil. Cada escala é medida por meio de Escala Likert de sete níveis (nunca - médio - muito, quantificados de 0 a 6 ). Os autores concluíram que, quanto maior a satisfação corporal, maior a satisfação sexual. Já no aspecto percepção do prazer sexual, as mulheres detalharam características, físicas e emocionais em relação à satisfação sexual, e em relação ao comportamento sexual, os homens preferem diversificar os parceiros sexuais, enquanto as mulheres preferem parceiros sexuais e afetivos fixos. Os homens e as mulheres menos liberais em relação à religiosidade tendem a gostar menos de sexo (Traen, Markovic, \& Kvalem, 2016; Cardoso, Sacomori, Sperandio, \& Krüger, 2012). No entanto, tal instrumento não apresentou evidências de validade e não foi desenvolvido com adaptação para o público de mulheres lésbicas.

Outro estudo importante foi realizado por Catão et al. (2010) que aplicou a Escala de Satisfação Sexual para Mulheres (SSS-W) de Meston e Trapnell (2005), em 20 mulheres em processos psicoterapêuticos, sem queixas sexuais e com idade média de 33 anos. A análise estatística apontou relação significativa entre os itens, com a avaliação de consistência interna adequada quanto aos níveis de precisão (alfa de Cronbach) de 0,91. O instrumento SSS-W 
se mostrou ferramenta promissora no contexto clínico para o auxílio das questões em relação à sexualidade.

Outra pesquisa interessada no fenômeno da satisfação sexual foi a de Monteiro et al. (2016), realizada com 225 mulheres grávidas, com idade entre 18 e 45 anos, no ambulatório da Faculdade de Maternidade da Universidade Federal do Rio Grande do Norte. Os autores investigaram a função sexual das mulheres por meio do Female Sexual Function Index (FSFI), que possui 19 questões que avaliam seis domínios da resposta sexual: desejo, excitação, lubrificação, orgasmo, satisfação e dor. O estudo apontou que a gravidez pode ser significativo para o desenvolvimento de disfunção sexual entre as mulheres grávidas, em que, 66,7\% mostraram risco de disfunção sexual, e as dimensões de desejo $(2,67)$, satisfação sexual $(2,71)$ e excitação sexual $(2,78)$ foram as mais afetadas. Contudo, o estudo apontou que a sexualidade das mulheres deve continuar a se desenvolver, levando em consideração que os desejos e a satisfação sexual dependem principalmente da condição psicológica e da aceitação da gravidez.

Assim, diante da discussão apresentada, pode-se perceber que "a satisfação sexual tem sido a variável da sexualidade examinada com mais frequência" (Carvalheira \& Leal, 2008, p. 4). Contudo, até bem pouco tempo, os estudos sobre a sexualidade de mulheres eram resultados de apreensões da sexualidade de homens, em que diante das inúmeras tentativas de se generalizar a sexualidade, não se pode assumir uma definição cientifica baseada em somente associações de aspectos tão imensamente distantes e artificiais. Desse modo, os instrumentos sobre sexualidade e satisfação sexual com mulheres lésbicas precisam ser desenvolvidos para que não se reduza a sexualidade a uma reprodução reguladora e normatizadora dos modos de pensar, viver e desejar em relação à sexualidade. Ou seja, percebe-se que a discussão sobre a sexualidade de mulheres lésbicas ainda é considerada socialmente um tabu e muito mais forte se expressa esse preconceito ao verificar que são restritos em contexto nacional os estudos sobre a sexualidade de mulheres lésbicas (Rich, 2012).

Faz-se necessário, portanto, a construção de um instrumento sobre a satisfação sexual que ofereça uma abordagem mais integrada e específica para mulheres lésbicas (Wainberg, Hutz, \& Stenert, 2009). Assim, baseado no Questionário de Identidade Corporal (QIC) aplicado em homens e mulheres no Brasil (Cardoso, Marinho, \& Pimentel, 2013), este estudo tem como objetivo a construção e a descrição das etapas da criação de um instrumento psicométrico e a descrição das evidências de validação do instrumento específico para a população lésbica.

\section{Método}

\section{Participantes da pesquisa}

A pesquisa foi desenvolvida de forma online, com uma amostra de 1.231 mulheres lésbicas, com idade entre 15 e 67 anos $(M=27,87 ; D P=9,10)$. Mulheres de todos os estados brasileiros responderam à pesquisa, em que 24,4\% (n =300) são de São Paulo; 16,7 ( $n=205)$ do Ceará; 10,8 $(\mathrm{n}=133)$ do Rio de Janeiro; $9,7(\mathrm{n}=$ 119) Minas Gerais e 7,9 $(n=97)$ do Rio Grande do Sul.

Cerca de $60,8 \%(n=311)$ das mulheres pesquisadas se reconhecem como brancas, $60,4 \%(n=744)$ são universitárias e tiveram a sua primeira relação sexual com uma mulher em torno dos 19 anos de idade. A média em relação à quando perceberam a sua orientação sexual, se deu entre os 15 anos de idade e a média de quando se assumiram socialmente lésbicas aconteceu em torno dos 18 anos.

\section{Instrumentos da pesquisa}

Foram aplicados dois instrumentos que serviram como ponto de partida para a criação do instrumento específico para mulheres lésbicas. O primeiro instrumento foi o questionário sociodemográfico especialmente elaborado para esse estudo, que continha questões acerca da idade, profissão, renda, e religiosidade. E o segundo instrumento foi o Questionário de Identidade Corporal (QIC) desenvolvido por Cardoso, Marinho \& Pimentel 
(2013) que possui três dimensões - sexualidade, corporeidade e motricidade em 10 subescalas, composta por 71 itens, mensurados a partir de uma Escala Likert de quatro níveis (nunca - pouco - médio - muito).

\section{Procedimentos de coleta e análise de dados}

Para a construção e a validação do instrumento psicométrico específico para a população lésbica foram realizadas cinco etapas: 1) Avaliação qualitativa dos instrumentos pelo grupo focal com experts; 2) Avaliação dos instrumentos pelas informantes-chave; 3) Análise por juízes-pares; 4) Análise Fatorial Exploratória (AFE); e 5) Análise Fatorial Confirmatória (AFC).

Os estudos qualitativos (estudos 1, 2 e 3) foram organizados a partir da formação de um grupo focal com 8 experts (1), em seguida foram realizadas as entrevistas com as informantes-chave (2), posteriormente foi realizada uma análise de juízes-pares (3) (Alves, Rabelo, \& Souza, 2014; Dias, 2000). O instrumento foi disponibilizado no Google Forms e divulgado em redes sociais digitais pelo período de três meses (fevereiro, março e abril) em 2017. A autora principal da pesquisa precisou entrar em mais de 160 grupos fechados e abertos no Facebook, Instagram e Whatsapp para poder acessar mulheres lésbicas de todos os estados brasileiros.

Para a avaliação da estrutura fatorial da Escala de Satisfação Corporal e Satisfação Sexual para Lésbicas foi realizado um procedimento de análise fatorial em duas etapas: exploratória e confirmatória. Os resultados da Análise Fatorial Exploratória (4) e os resultados da Análise Fatorial Confirmatória (5) foram conduzidos com o estimador Weighted Least Squares Means and Variance Adjusted no software Mplus versão 7.11. Para tanto, o banco de dados foi dividido ao meio aleatoriamente. Inicialmente, foi realizada AFE considerando todos os 30 itens da escala, utilizando a primeira metade do banco. Em seguida, o modelo proposto na AFE foi testado em uma AFC. Diferentemente da AFE que, de modo livre, mostra uma estrutura e as suas correlações entre as variáveis e indicadores de ajustamentos do mo- delo, a AFC da ESCSS-Lésbicas já assume um caráter sistemático, em que se deve confirmar a dimensionalidade das correlações entre os itens, a reorganização da escala e a renomeação dos fatores a partir dos itens que se correlacionam (Dancey \& Reidy, 2013; Cassepp-Borges \& Teodoro, 2007; Cassepp-Borges \& Pasquali, 2012; Andrade, Garcia \& Cassepp-Borges, 2013; Brown, 2006).

Ambas as análises foram conduzidas com o estimador Weighted Least Squares Means and Variance Adjusted (WLSMV) para controlar para a natureza categórica dos itens da escala tipo Likert, no software Mplus versão 7.11. Como índices de ajuste foram analisados o Comparative Fit Index (CFI), o Tucker-Lewis Index (TLI), o Root Mean Square Error of Approximation (RMSEA), com um intervalo de confiança de $90 \%$, e o Standardized Root Mean Square Residual (SRMR). Valores do CFI e do TLI próximos a 0,90 representam ajuste aceitável, e acima de 0,95 representam ajuste ótimo (Marsh, Balla, \& Hau, 1996; Rigdon, 1996). Valores do RMSEA e do SRMR próximos a 0,08 representam ajuste aceitável, e abaixo de 0,05 ajuste ótimo (Brown, 2006; Hu, \& Bentler, 1999). Conforme pode ser observado na Tabela 1, os resultados da AFE indicam que somente a partir da extração de cinco fatores a totalidade dos índices da estrutura fatorial da escala se aproxima de índices de ajustes aceitáveis.

\section{Resultados e discussões}

\section{Avaliação do instrumento original pelo grupo focal com experts}

Foram consideradas experts pessoas com idade acima de 18 anos, pesquisadores e/ou profissionais, que estão vinculados às discussões de gênero, sexualidade e lesbianidades, seja a partir de organizações não governamentais, associações, grupos de militância, grupos de estudos em universidades e pós-graduações. O grupo focal foi composto por 8 experts no tema proposto, que se reuniram em 4 encontros com duração mínima de 2 horas, para a realização da leitura discutida e siste- 
mática do instrumento. $\mathrm{O}$ grupo focal se configurou como etapa decisiva para a criação e a validação do instrumento (Pascoal, Narciso, Pereira, \& Ferreira, 2013; Marshall, 1996). Os encontros foram filmados por três câmeras filmadoras, gravados em áudios e contaram com a presença de uma assistente de pesquisa para a transcrição das observações em sala.

O grupo de experts sugeriu algumas alterações no instrumento original QIC; a) retirada de duas dimensões propostas pelo questionário original - motricidade $\mathrm{e}$ corporeidade relacionada a infância; b) a retirada de 3 subescalas do instrumento original - identidade infantil, orientação sexual e comportamento sexual; c) alterações de linguagens em alguns itens, como por exemplo, no item que questiona "quanto eu gosto do meu pênis ou vagina"; d) a inclusão de outro item em relação "ao quanto eu gostaria de fazer mudança de sexo".

O grupo de experts defendeu que o instrumento precisava possuir especificidades que questionassem a sexualidade de mulheres lésbicas em todas as etapas da vida. E para não perder o sentido semântico para o contexto da população pesquisada - mulheres lésbicas - o instrumento deveria contemplar no seu título referência à sexualidade lésbica.

O objetivo do grupo focal foi realizar a validade de conteúdo, que se refere à avaliação da clareza da linguagem específica para o grupo de mulheres lésbicas. A pertinência prática do assunto pesquisado foi avaliada considerando a materialização do cotidiano, relevância e a dimensão teórica em que "o teste foi submetido à avaliação de juízes, que deveriam indicar se o item era essencial ao teste" (Pacico \& Hutz, 2015, p. 75). Paralelamente, foram constatadas as evidências de validade de constructo do instrumento, que determinou a dimensão que a escala satisfação corporal e satisfação sexual se propôs a avaliar (Borsa, Damásio, \& Bandeira, 2012; Cassepp-Borges, Balbinotti, \& Teodoro, 2010; Gjersing, Caplehorn, \& Clausen, 2010; Pasquali,1997; Ross \& Rosser, 1996).

\section{Avaliação do instrumento pelas informantes-chave}

Foram consideradas informantes-chave, 6 mulheres lésbicas com idade mínima de 45 anos de idade, contactadas uma a uma, em que foi discutido item a item do instrumento. As mulheres ficaram com o instrumento por cinco dias e enviaram um feedback por e-mail.

Após o recebimento dosfeedbacks, decidiu-se por: a) retirar 2 subescalas e b) repensar o tamanho do instrumento, pois possuía escala que fugia da temática da pesquisa. De uma forma geral, as informantes-chave afirmaram que o instrumento possuía questões sobre a identidade infantil com perguntas sobre "o quanto eu queria ser bailarina ou o quanto eu gostava de jogar videogame", questões sobre o comportamento sexual e a orientação sexual que excediam a finalidade da pesquisa.

Considerando as mudanças propostas a partir do grupo focal e das informantes-chave, foram excluídas do instrumento original cinco subescalas que fugiam o escopo da pesquisa e foram realizadas alterações semânticas no corpo do texto. Desse modo, tendo em vista todas as alterações realizadas, os autores sugeriram a nomeação do instrumento: Escala de Satisfação Corporal e Satisfação Sexual para Lésbicas (ESCSS - Lésbicas).

\section{Análise por juízes-pares}

Foram convidados quatro pesquisadores na temática de gênero e sexualidade, aqui denominados juízes-pares para comporem o corpo de análise do instrumento. Após a avaliação do grupo de experts e do grupo de informantes-chave, a Escala de Satisfação Corporal e Satisfação Sexual para Lésbicas (ESCSS-Lésbicas), tipo Likert ficou gradativa de 0 a 6 , divididos em 5 subescalas. Os juízes-pares leram individualmente e minunciosamente o instrumento e, de forma consensual confirmaram a possibilidade de aplicabilidade do instrumento em mulheres lésbicas. 
Análise Fatorial Exploratória e Análise Fatorial Confirmatória da Escala de
Satisfação Corporal e Satisfação Sexual para Lésbicas (ESCSS - Lésbicas)

Tabela 1. Índices de ajuste dos modelos na AFE e na AFC da Escala de Satisfação Corporal e Satisfação Sexual para Lésbicas (ESCSS - Lésbicas) utilizando Metades Aleatórias da Amostra

\begin{tabular}{|c|c|c|c|c|c|}
\hline $\operatorname{AFE}[n=631]$ & $\chi^{2}(\mathrm{df})$ & CFI & TLI & RMSEA $[90 \%$ CI $]$ & SRMR \\
\hline 1 Fator & $9979.21(560)$ & .664 & .644 & $.163[.160-.166]$ & .158 \\
\hline 2 Fatores & $6732.96(526)$ & .779 & .750 & $.137[.134-.140]$ & .110 \\
\hline 3 Fatores & $4139.54(493)$ & .870 & .843 & $.108[.105-.111]$ & .081 \\
\hline 4 Fatores & $3114.17(461)$ & .905 & .878 & $.096[.092-.099]$ & .067 \\
\hline 5 Fatores & $2538.40(430)$ & .925 & .896 & $\mathbf{. 0 8 8}[.085-.091]$ & .059 \\
\hline $\mathrm{AFC}[n=600]$ & $\chi^{2}(\mathbf{d f})$ & CFI & TLI & RMSEA [90\% CI] & \\
\hline 5 Fatores & $1833.12(393)$ & .949 & .944 & $.078[.0750 .082]$ & 1.740 \\
\hline
\end{tabular}

Nota. CFI: Comparative Fit Index; TLI: Tucker-Lewis Index; RMSEA [90\% CI]: Root Mean Square Error of Approximation with 90\% Confidence Interval; SRMR: Standardized Root Mean Square Residual

Pelos resultados da AFE, foram excluídos os itens 6, 8, 10 e 11 do fator Satisfação Corporal e o item 5 do fator Disposição Sexual por apresentarem cargas mais baixas que 0,30, fazendo com que a ESCSS-Lésbicas ficasse com 30 itens no total. Os resultados da AFC, com o modelo de cinco fatores proposto na AFE após a exclusão dos itens relatados acima, apresentaram índices de ajuste aceitáveis [CFI $=, 925$; TLI $=, 896$; RMSEA $=, 059]$. Os resultados da AFC também indicaram uma correlação entre erros entre os itens 5 e 7 da escala de Intimidade Corporal e entre os itens 1 e 2 da escala de Satisfação Sexual, indicando que esses itens compartilham muita variância, possivelmente sugerindo-se modificar a escrita deles ou uni-los em cada fator.

Por fim, foi realizada uma análise do teste de diferença qui-quadrado $\left(\Delta \chi^{2}\right)$ para verificar se os índices de qualidade do modelo de cinco fatores eram significativamente diferentes dos índices do modelo de quatro fatores. Os resultados do teste demonstraram que o modelo de cinco fatores apresentou índices sig- nificativamente melhores de ajuste do que o modelo de quatro fatores $(\Delta \chi 2=86.38(4), \mathrm{p}<0,001)$.

A Figura 1 apresenta, as cargas fatoriais dos itens em cada um dos cinco fatores conforme a AFE do modelo de cinco fatores e conforme a AFC do modelo de cinco fatores. Pode ser observado, ainda, que a carga fatorial de cada item confirmou o modelo teórico do refinamento do instrumento e apresenta agora uma escala mais específica para a aplicação com o público estudado. 


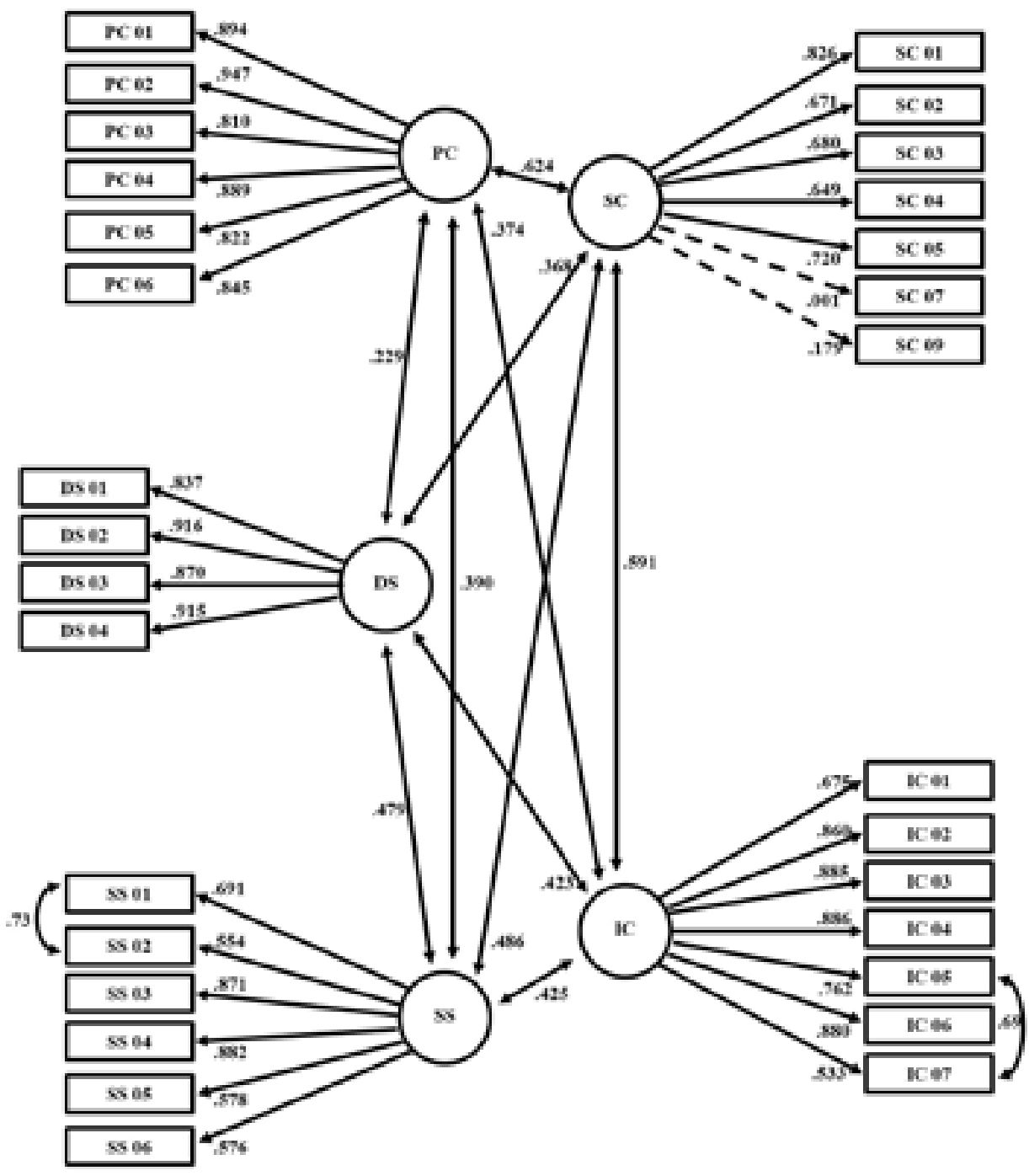

Figura 1. Diagrama da Escala de Satisfação Corporal e Satisfação Sexual para Lésbicas (ESCSS-Lésbicas) com as Cargas Fatoriais dos itens da amostra brasileira.

Nota: Fatores - (PC) Percepção Corporal; (SC) Satisfação Corporal; (IC) Intimidade Corporal; (DS) Disposição Sexual e (SS) Satisfação Sexual. *As cargas fatoriais estão ao lado de cada item. **Os itens 7 e 9 da dimensão Satisfação Corporal foram invertidos.

Pode-se perceber na Figura 1 que os fatores 1 (PC), 3 (IC) e 5 (SS) foram os únicos fatores que apresentaram todos os índices aceitáveis nas AFCs unidimensionais dos fatores originais isolados. O fator 2 (SC) e 4 (DS) apresentaram resultados mistos, enquanto o fator 2 apresentou pior ajuste.

A escala ESCSS-Lésbicas validada possui 5 subescalas e 30 itens: Percepção Corporal (PC) com 6 itens $(\alpha=0,92)$; Satisfação Corporal (SC) com 7 itens $(\alpha=$ 0,77); Intimidade Corporal (IC) com 7 itens $(\alpha=0,88$ ); Disposição Sexual (DS) com 4 itens ( $\alpha=0,72$ ) e Satisfação
Sexual (SS) com 6 itens $(\alpha=0,78)$. A confiabilidade interna (alfa de Cronbach) da escala total é de 0,90 ).

Modelos unidimensionais considerando cada um dos cinco fatores originais isoladamente foram também testados para avaliar qual(is) fator(es) obteve(tiveram) ajuste bom ou ruim. Concluiu-se então, que o modo integral da escala é a forma mais adequada para aplicação da ESCSS-Lésbicas, por existir correlação entre as subescalas e refuta-se o uso das subescalas sozinhas por não considerarem as correlações e apresentarem ajustes ruins. 


\section{Considerações finais}

A partir da percepção de ausência de instrumentos psicométricos específicos para a população lésbica em contexto nacional, este estudo teve como objetivo a construção e a descrição das etapas da criação de um instrumento psicométrico e a descrição das evidências de validação do instrumento específico para a população lésbica, aplicado em uma amostra de 1.231 mulheres lésbicas e brasileiras. O processo que incluiu as etapas de criação e validação envolveu técnicas psicométricas no intuito de encontrar e apresentar um instrumento robusto para avaliar a satisfação corporal e a satisfação sexual em mulheres lésbicas (Borsa, Damásio, \& Bandeira, 2012; Gjersing, Caplehorn, \& Clausen, 2010).

Compreende-se que a validação de uma escala sobre satisfação corporal e sexual específica para mulheres lésbicas, deve levar em consideração a complexidade que perpassa todo o contexto socioemocional vivido por essas mulheres. Para além da expectativa corporal, muitas vezes, mulheres lésbicas em contexto brasileiro escutam o quanto a sua sexualidade é um desperdício corporal e de vida, por não serem heterossexuais. Isso é, lésbicas no Brasil e em contexto ocidental, lidam cotidianamente com a expectativa-obrigatoriedade de possuírem corpos demarcados pela feminilidade e pela compulsória heterossexualidade (Rich, 2012). Contudo, percebe-se que o contexto é perpassado por demarcações homofóbicas e nesse sentido, a ESCSSLésbicas se preocupa na percepção que essas mulheres possuem sobre os próprios corpos e sua sexualidade tanto em contexto social como em contextos clínicos.

A saúde sexual da mulher lésbica deve ser um fator importante a ser considerado nos sistemas nacionais de saúde, na formação de profissionais e serviços que apresentem uma linguagem inclusiva (Carvalheira \& Leal, 2008; Sprecher et al., 2006; Wainberg, Hutz, \& Stenert, 2009). Para tanto, espera-se que os processos educacionais, sociais e jurídicos ampliem as suas redes de estudos acerca da corporeidade e de diferentes expressões da sexuali- dade humana, implicando diretamente na promoção da qualidade de vida biopsicossocial de lésbicas no Brasil (Catão et al., 2010; Henderson, Lehavot, \& Simoni, 2009).

Este estudo possibilitou a construção de um instrumento que investiga a sexualidade lésbica que não existia em contexto brasileiro, contemplou a participação da voz das mulheres de múltiplas formas, como por exemplo, no grupo focal com experts, as entrevistas com as informantes-chave e uma amostra que abrangeu todos os estados brasileiros. Ou seja, o percurso do estudo visibilizou a expressão da sexualidade de mulheres lésbicas, e resultou no instrumento final - ESCSSLésbicas, que apresentou suporte válido e fidedigno para a aplicação em mulheres lésbicas.

Algumas limitações deste estudo devem ser listadas como, por exemplo, o procedimento de coleta online, que apontou um perfil geográfico e social de mulheres que necessariamente precisavam ter acesso às tecnologias para responderem a pesquisa. Outra questão a ser mencionada, é a cultura social que está atrelada às novas gerações e a relação mais íntima com as novas tecnologias, fenômeno que pode ter influenciado a maioria de mulheres entre 20 e 40 anos a participar. Uma sugestão para próximos estudos é a possibilidade de um estudo tipo longitudinal, que considere as trajetórias de vida e possibilite a ampliação das discussões aqui iniciadas. Recomenda-se que futuras pesquisas sobre satisfação corporal e satisfação sexual considerem esses aspectos apontados para obterem resultados que visibilizem a sexualidade de mulheres lésbicas no Brasil.

\section{Referências}

Alves, P. C., Rabelo, M. C., \& Souza, I. M. (2014). Hermenêutica-fenomenológica e compreensão nas ciências sociais. Sociedade e Estado, 29(1), 181-198. https://doi.org/10.1590/S0102-69922014000100010 
American Psychological Association [APA]. (2008) Answers to your questions: For a better understanding of sexual orientation and homosexuality Washington, DC: Author Disponível online a 27 de Abril de 2010 em http://www apa org/pi/about/newsletter/2008/04/ brochureupdate aspx.

Andrade, A. L. D., Garcia, A., \& Cassepp-Borges, V. (2013). Evidências de validade da Escala Triangular do Amor de Sternberg-Reduzida (ETAS-R). PsicoUSF, 18(3), 501-510. https://doi.org/10.1590/S1413$\underline{82712013000300016}$

Borsa, J. C., Damásio, B. F., \& Bandeira, D. R. (2012). Adaptação e Validação de Instrumentos Psicológicos entre Culturas. Paidéia, 22(53), 423-432. https://doi. org/10.1590/S0103-863X2012000300014

Brown, T. A. (2006). Confirmatory factor analysis for applied research. New York: The Guilford Press.

Cardoso, F. L., (1994). Noções de corporeidade - de quem para quem? Motrivivência, 5, 170-177.

Cardoso, F. L., Savall, A. C., Sabbag, S., Mendes, A. K., \& Beltrame, T. S. (2009). Implicações do conhecimento corporal no comportamento sexual. Revista Brasileira de Educação Física e Esporte, 23(4), 345-354. https:// doi.org/10.1590/S1807-55092009000400004

Cardoso, F. L., Sacomori, C., Sperandio, F. F., \& Krüger, A. P. (2012). Satisfação corporal em acadêmicos de Educação Física: proposta de um questionário. Motriz Revista Educação Física (Impr.), 18(1), 63-71. https:// doi.org/10.1590/S1980-65742012000100007

Cardoso, F. L., Marinho, A., \& Pimentel, G. G. A. (2013). Questões de gênero em universitáriospraticantes de esportes de aventura. Journal of Physical Education, 24(4), 597-608.

Cassepp-Borges, V., \& Teodoro, M. L. (2007). Propriedades psicométricas da versão brasileira da Escala Triangular do Amor de Sternberg. Psicologia: Reflexão e Crítica, 20(3), 513-522. https://doi.org/10.1590/ S0102-79722007000300020

Cassepp-Borges, V., Balbinotti, M. A. A., \& Teodoro, M. L. M. (2010). Tradução e validação de conteúdo: uma proposta para a adaptação de instrumentos. In L. Pasquali (Org.), Instrumentação psicológica: Fundamentos e práticas (pp. 506-520) . Porto Alegre: Artmed.
Cassepp-Borges, V. \& Pasquali, L. (2012). Estudo nacional dos atributos psicométricos da Escala Triangular do Amor de Sternberg. Paidéia, 22(51), 21-31. https:// doi.org/10.1590/S0103-863X2012000100004

Catão, E., Rodrigues Jr, O. M., Viviani, D. H., Finotelli Jr, I., \& Silva, F. R. (2010). Escala de Satisfação Sexual para Mulheres: Tradução, adaptação em estudo preliminar com amostra clínica. Boletim de Psicologia, 60(133), 181-190.

Carvalheira, A. A., \& Leal, I. (2008). Os determinantes da satisfação sexual feminina: um estudo português. Revista Internacional de Andrología, 6(1), 3-7. https:// doi.org/10.1016/S1698-031X(08)72559-7

Cerqueira-Santos, E., Winter, F. S., Salles, L. A., Longo, J. L., \& Teodoro, M. L. M. (2007). Contato interpessoal e crenças sobre homossexualidade: Desenvolvimento de uma escala. Interação em Psicologia, 11(2), 221-229. doi: 10.5380/psi.v11i2.6639 https://doi.org/10.5380/psi. v11i2.6639

Dancey, C. P., \& Reidy, J. (2013). Estatística sem matemática para psicologia. RS: Penso Editora.

Derogatis, L. R., \& Melisaratos, N. (1979). The DSFI: A multidimensional measure of sexual unctioning. Journal of sex \& marital therapy, 5(3), 244-281. https://doi.org/10.1080/00926237908403732

Dias, C. A. (2000). Grupo focal: técnica de coleta de dados em pesquisas qualitativas. Informação \& Sociedade: Estudos, 10(2), 1-12.

Duby, G. \& M. Perrot. (1998). História das Mulheres: $O$ século $X X$. Porto: Afrontamento.

Fernandes, J., Barroso, K., Assis, A., \& Pocahy, A. F. (2015). Gênero, sexualidade e envelhecimento: uma revisão sistemática da literatura. Rev. Clínica \& Cultura, 4(1), 14-28.

Fernandes, J. (2012). Mulher e política: A experiência vivida de mulheres no exercício do poder político. (Dissertação de mestrado Universidade de Fortaleza). Recuperado de http://bdtd.ibict.br/vufind/Record/ UFOR d14678194e9491281cf9342066402ald.

Furlanetto, S. H. T., \& Rodrigues Jr., O. M. (1996). A satisfação sexual da mulher adulta. Revista Brasileira de Sexualidade Humana, 7 (1), 131143. 
Gato, J., Fontaine, A. M., \& Leme, V. B. R. (2014). Validação e Adaptação Transcultural da Escala Multidimensional de Atitudes Face a Lésbicas e a Gays. Psicologia: Reflexão e Crítica, 27(2), 257-271. https://doi.org/10.1590/1678-7153.201427206

Gjersing, L., Caplehorn, J. R., \& Clausen, T. (2010). Cross-cultural adaptation of research instruments: language, setting, time and statistical considerations. BMC medical research methodology, 10(1), 13. https:// doi.org/10.1186/1471-2288-10-13

Haraway, D. (2004). Gênero para um dicionário marxista. Cadernos Pagu, 22, 201-246. https://doi.org/10.1590/ $\underline{\text { S0104-83332004000100009 }}$

Henderson, A. W., Lehavot, K., \& Simoni, J. M. (2009). Ecological models of sexual satisfaction among lesbian/ bisexual and heterosexual women. Archives of sexual behavior, 38(1), 50-65. https://doi.org/10.1007/s10508-008-9384-3

Hu, L., \& Bentler, P. M. (1999). Cutoff criteria for fit indexes in covariance structure analysis: Conventional criteria versus new alternatives. Structural Equation Modeling: A Multidisciplinary Journal, 6, 1-55. https:// doi.org/10.1080/10705519909540118

Hudson W. W. (1998). Index of Sexual Satisfaction. In Davis, Yarber, Bauserman, Schreer, Davis. Handbook of sexuality-related measures. (pp. 512-513). Thousand Oaks, CA: Sage.

Lee, H.; Damhorst, M. L.; Ogle, J. P. (2009). Body satisfaction and attitude theory: linkages with normative compliance and behaviors undertaken to change the body. Family and Consumer Sciences Research Journal, 37(4), 466-488. https://doi.org/10.1177/1077727X09333165

Lingiardi, V., Baiocco, R., \& Nardelli, N. (2012). Measure of internalized sexual stigma for lesbians and gay men: A new scale. Journal of homosexuality, 59(8), 1191-1210.

LoPiccolo, J., \& Steger, J. C. (1974). The Sexual Interaction Inventory: A new instrument for assessment of sexual dysfunction. Archives of sexual behavior, 3(6), 585-595. https://doi.org/10.1007/BF01541141
Marsh, H. W., Balla, J. R., \& Hau, K.-T. (1996). An evaluation of incremental fit indices: A clarification of mathematical and empirical properties. In G. A. Marcoulides \& R. E. Schumacker (Eds.), Advanced structural equation modeling: Issues and techniques. Mahwah, NJ: Erlbaum.

Marshall, M. N. (1996). The Key Informant Technique. Family Practice, 13(1), 92-97.

Meston, C. \& Trapnell, P. (2005). Development and validation of a fivefactor sexual satisfaction and distress scale for women: The Sexual Satisfaction Scale for Women (SSSW). Journal of Sexual Medicine, 2(1), 6681. https://doi.org/10.1111/j.1743-6109.2005.20107.x

Monteiro, M. N., Lucena, E. E. S, Cabral, P. U., Queiroz Filho, J., Queiroz, J., \& Gonçalves, A. K. (2016). Prevalence of Sexual Dysfunction among Expectant Women. Revista Brasileira de Ginecologia e Obstetrícia, 38(11), 559-563. https://doi.org/10.1055/s-0036-1594306

Nogueira, C., \& Oliveira, J. M. (2010). Um olhar da psicologia feminista crítica sobre os direitos humanos de pessoas GLBT- Estudo sobre a discriminação em função da orientação sexual e da identidade de género. Lisboa, Portugal.

O'Murray, S (2002) The Comparative Sociology of Homosexualities In D Richardson \& S Seidman (Eds). Handbook of Lesbian and Gay Studies, London: Sage. https://doi.org/10.4135/9781848608269.n7

Pacico, J. C., \& Hutz, C. S. (2015). Validade. In C. S. Hutz, D. R. Bandeira \& C. M. Trentini. (Orgs). Coleção Avaliação Psicológica. Porto Alegre, Artmed.

Pascoal, P. M., Narciso, I. D. S. B., Pereira, N. M., \& Ferreira, A. S. (2013). Processo de validação da Global Measure of Sexual Satisfaction em três amostras da população portuguesa. Psicologia: Reflexão e Crítica, 26(4), 691-700.

Pasquali, L. (1997). Psicometria: teoria e aplicações. Brasília: Universidade de Brasília.

Pechorro, P., Diniz, A., \& Vieira, R. (2009). Satisfação sexual feminina: Relação com funcionamento sexual e comportamentos sexuais. Análise psicológica, 27(1), 99-108.

Pinney, E. M., Gerrard, M., \& Denney, N. W. (1987). The Pinney Sexual Satisfaction Inventory. Journal of Sex Research, 23(2), 233-251. https://doi. org/10.1080/00224498709551359 
Rich, A. (2012). Heterossexualidade compulsória e existência lésbica. Bagoas-Estudos gays: gêneros e sexualidades, 4(05).

Rigdon, E. E. (1996). CFI versus RMSEA: A comparison of two fit indexes for structural equation modeling. Structural Equation Modeling: A Multidisciplinary Journal, 3(4), 369-379. https://doi. org/10.1080/10705519609540052

Ross, M. W., \& Rosser, B. R. S. (1996). Measurement and Correlates of Internalized Homophobia: a Factor Analytic Study. Journal of Clinical Psychology, 52(1), 1521 https://doi.org/10.1002/(sici)1097-4679(199601)52:1\%3C15::aid-jclp2\%3E3.0.co;2-V

Shidlo, A. (1994). Internalized homophobia: Conceptual and empirical issues in measurement. In Some of the information in this chapter was presented at the meeting of the American Psychological Assn, New York, 1987. Sage Publications, Inc.

Sprecher, S., Christopher, F. S., Cate, R., Vangelisti, A. L., \& Perlman, D. (2006). Sexuality in close relationships. In The cambridge handbook of personal relationships (pp. 463-482). New York: Cambridge University Press. https://doi.org/10.1017/cbo9780511606632.026

Traen, B., Markovic, A., \& Kvalem, I. L. (2016). Sexual satisfaction and body image: a cross-sectional study among Norwegian young adults. Sexual and Relationship Therapy, 31(2), 123-137. https://doi. org/10.1080/00224498709551359

Wainberg, L.; Hutz, C.S., \& Stenert, F. (2009). Construção de instrumento de medida de satisfação sexual. Resumos do IV Congresso Brasileiro de Avaliação Psicológica, Campinas, 177.

Whitley M. P. (1998) Sexual Satisfaction Inventory. In Davis CM, Yarber WL, Bauserman R, Schreer G, Davis SI, (Eds). Handbook of sexuality-related measures. (pp.519-521). Thousand Oaks, CA: Sage.

Recebido em: 19/2/2018.

Aprovado em: 12/8/2019.

Publicado em: $\mathrm{xx} / \mathrm{x} / \mathrm{xxxx}$

Endereço para correspondência: Juliana

Fernandes-Eloi (Rua César Fonseca, 455, Apto, 802.
CEP: 60192-260, Cocó, Fortaleza; (85)9.9940-9222;

julianafernandeseloi@gmail.com).

\section{Nome: Juliana Fernandes-Eloi}

E-mail: julianafernandeseloi@gmail.com

Titulação Acadêmica: Doutorado em Psicologia

Afiliação Institucional: Universidade de Fortaleza -

UNIFOR; Estácio do Ceará; Unichritus

\section{Nome: Luciana Maria Maia}

E-mail: lumariamaia@hotmail.com

Titulação Acadêmica: Doutorado em Psicologia

Afiliação Institucional: Universidade de Fortaleza -

UNIFOR

\section{Nome: Elder Cerqueira-Santos}

E-mail: eldercerqueira@gmail.com

Titulação Acadêmica: Doutorado em Psicologia

Afiliação Institucional: Universidade Federal de Sergipe - UFS 